\title{
Self-similar solutions for a class of non-divergence form equations
}

\author{
Chunhua Jin and Jingxue Yin
}

\begin{abstract}
In this paper, we study the self-similar solutions for a non-divergence form equation of the form

$$
u(x, t)=(t+1)^{-\alpha} f\left((t+1)^{\beta}|x|^{2}\right) .
$$

We first establish the existence and uniqueness of solutions $f$ with compact supports, which implies that the self-similar solution is shrink. On the basis of this, we also establish the convergent rates of these solutions on the boundary of the supports. On the other hands, we also consider the convergent speeds of solutions, and compare which with Dirac function as $t$ tends to infinity.
\end{abstract}

Keywords. Self-similar solution, Non-divergence, Convergent rates.

\section{Introduction}

This paper is concerned with the self-similar solutions for the following degenerate and singular parabolic equation in non-divergence form

$$
\frac{\partial u}{\partial t}=u^{m} \operatorname{div}\left(|\nabla u|^{p-2} \nabla u\right)
$$

where $m \geq 1, p>1$. This equation comes from many physical problems such as dispersal mechanisms on species survival, plasma physics, damage mechanics, curve shortening flow and so on, see for example [1-5].

Equations of the form (1.1) have some special characteristics distinguished from those in divergence form. Different from the finite speed of propagation of disturbances for porous medium equation, or the infinite speed of propagation of disturbances for heat equation, the support of solutions of the

This work is partially supported by the National Science Foundation of China, and partially supported by the Specialized Research Fund for the Doctoral Program of High Educational of China. 
equation (1.1) will never expand. Another important feature of this kind of equations is the non-uniqueness of solutions, which has been discovered by Dal Passo and Luckhaus [6], for the special case $m=1$ and $p=2$. Indeed, for any $T>0$, they have constructed a weak solution with extinction time $T$. However, a so-called maximal solution with constant support is uniquely determined by the initial datum. It is easy to verify that if $u(x, t) \not \equiv 0$ is a solution of the equation (1.1), then by the scaling,

$$
u_{\lambda}(x, t)=\lambda^{\alpha} u\left(\lambda^{-\beta} x, \lambda t\right), \quad \beta \geq 0, \quad \alpha=\frac{1+\beta p}{m+p-2}
$$

for any $\lambda>0$, are solutions of the Eq. (1.1). Specially, take $\lambda=\frac{1}{t}$, then $u_{\lambda}(x, t)=t^{-\alpha} f\left(t^{\beta} x\right)$, which is called self-similar solution. Self-similar solutions often play important roles in the theory of regularity and asymptotic stability of nonlinear problems, the importance of looking for self-similar singularities of equations has been recognized by Leray [7] as early as 1934. Since then, the topic on the self-similar solution has attracted a great deal of attention. See for example [8-14].

Generally speaking, the study on the self-similar solutions mainly restrict on the following two types: the forward self-similar solution and the backward self-similar solution

$$
\begin{array}{ll}
u(x, t)=(t+\tau)^{-\alpha} f(\eta), & \eta=(t+\tau)^{\beta} x, \\
u(x, t)=(\tau-t)^{-\alpha} f(\eta), & \eta=(\tau-t)^{\beta} x .
\end{array}
$$

In the present paper, we are going to search a family of forward self-similar solutions, radially symmetric in $x$, of the form

$$
u(x, t)=(t+1)^{-\alpha} f(\eta)
$$

with same initial datum, where $\eta=(t+1)^{\beta}|x|^{2}, \beta \geq 0, \alpha=\frac{2+p \beta}{2(m+p-2)}$. Through a simple calculation, we see that $u(x, t)$ is a self-similar solution of the equation (1.1) if and only if $f$ satisfies that

$$
2^{p} \eta^{p / 2} f^{m}\left(\left|f^{\prime}\right|^{p-2} f^{\prime}\right)^{\prime}+(N+p-2) 2^{p-1} \eta^{(p-2) / 2} f^{m}\left|f^{\prime}\right|^{p-2} f^{\prime}-\beta \eta f^{\prime}+\alpha f=0 .
$$

Let $h=\eta^{p / 2}\left|f^{\prime}\right|^{p-2} f^{\prime}$. Then the above equation is transformed into

$$
\left\{\begin{array}{l}
f^{\prime}=\eta^{-\frac{p}{2(p-1)}}|h|^{q-2} h, \\
h^{\prime}=\frac{2-N}{2 \eta} h+2^{-p} \beta \eta^{(p-2) / 2(p-1)} f^{-m}|h|^{q-2} h-\frac{\alpha}{2^{p}} f^{1-m},
\end{array}\right.
$$

where $q$ is the dual number of the constant $p$, that is $\frac{1}{p}+\frac{1}{q}=1$. Due to the radial symmetry property of the solution, we have $f^{\prime}(0)=0$. In what follows, we consider the system (1.4) with the following initial value conditions

$$
\left\{\begin{array}{l}
f(0)=A, \quad(A>0), \\
h(0)=0 .
\end{array}\right.
$$

The purpose of this paper is to search solutions with compact support of the initial value problem (1.4) and (1.5). That is, there exists a constant $\gamma>0$, such that 
(i) $\quad f(\eta)>0$ for any $\eta \in[0, \gamma)$, and $f(\gamma)=0$;

(ii) $f$ is monotone decreasing on $[0, \gamma]$ and $f, h$ are right continuous at 0 and satisfying the system (1.4) in the classical sense, and $f$ is left continuous at $\gamma$.

We see that if $f$ is a solution of (1.4)-(1.5), and satisfies (i) and (ii), let $f(\eta)=0$ for $\eta>\gamma$, from [15], the $u(x, t)$ defined by (1.2) is a weak solution of the equation (1.1) in $\mathbb{R}^{N} \times \mathbb{R}$.

We establish the existence of a unique solution with compact support for every $\beta \geq 0$. One will see that if $\beta=0$, the supports of solutions of the equation (1.1) remains constant in time, while if $\beta>0$, the supports keep shrinking and asymptotically go to a single point as $t \rightarrow \infty$. These results imply that the Eq. (1.1) admits infinite non-extinction solutions with compact supports for same initial data, which is different from the results in [6], where they decided the non-uniqueness of solutions is rooted in the existence of infinite extinction solutions. Moreover, on the basis of the established existence of solutions with compact support, we also discuss the convergent speeds of the solutions going to zero. At the same time, we also investigate the singularities of the reciprocal of solutions, and compare which with the Dirac function in some sense as $t$ tends to infinity. In addition, the non-existence of self-similar solutions without compact support also be discussed.

\section{Preliminaries}

To show the existence of solutions, we consider the corresponding approximate problem, that is the system (1.4) with the following initial conditions

$$
\left\{\begin{array}{l}
f(\varepsilon)=A, \quad(A>0), \\
h(\varepsilon)=0 .
\end{array}\right.
$$

By the theory of ordinary differential equations, the local existence of solutions for the problem (1.4), (2.1) is easy to be obtained. Furthermore, we have the following

Proposition 2.1. Let $\left(f_{\varepsilon}, h_{\varepsilon}\right)$ be a pair of solutions of the problem (1.4), (2.1). Then there exists $\gamma_{\varepsilon}$ with

$$
0<\gamma_{\varepsilon}<\left(2^{\frac{p}{N(p-1)}} \varepsilon^{\frac{p}{2(p-1)}}+\frac{p}{2(p-1)}\left(\frac{N 2^{p}}{\alpha}\right)^{\frac{1}{p-1}} A^{\frac{m+p-2}{p-1}}\right)^{\frac{2(p-1)}{p}}
$$

such that $f_{\varepsilon}\left(\gamma_{\varepsilon}\right)=0$ and $f_{\varepsilon}(\eta)>0, h_{\varepsilon}(\eta)<0$ for any $\eta \in\left(\varepsilon, \gamma_{\varepsilon}\right)$.

Proof. Since $\left(f_{\varepsilon}, h_{\varepsilon}\right)$ is a pair of solutions of the problem (1.4), (2.1), we have

$$
\left\{\begin{array}{l}
h_{\varepsilon}(\eta)=-\frac{\alpha}{2^{p}} \int_{\varepsilon}^{\eta} f_{\varepsilon}^{1-m}(s) \exp \left\{\int_{s}^{\eta} \frac{\beta}{2^{p}} \tau^{\frac{p-2}{2(p-1)}} f_{\varepsilon}^{-m}(\tau)\left|h_{\varepsilon}\right|^{q-2}-\frac{N-2}{2 \tau} d \tau\right\} d s, \\
f_{\varepsilon}(\eta)=A+\int_{\varepsilon}^{\eta} s^{-\frac{p}{2(p-1)}}\left|h_{\varepsilon}\right|^{q-2} h_{\varepsilon} d s .
\end{array}\right.
$$


Suppose to the contrary, for any $\eta>\varepsilon$,

$$
f_{\varepsilon}(\eta)>0, \quad h_{\varepsilon}(\eta)<0 .
$$

Noticing that

$$
\begin{aligned}
-h_{\varepsilon}(\eta) & =\frac{\alpha}{2^{p}} \int_{\varepsilon}^{\eta} f_{\varepsilon}^{1-m}(s) \exp \left\{\int_{s}^{\eta} \frac{\beta}{2^{p}} \tau^{\frac{p-2}{2(p-1)}} f^{-m}(\tau)\left|h_{\varepsilon}\right|^{q-2}-\frac{N-2}{2 \tau} d \tau\right\} d s \\
& \geq \frac{\alpha}{2^{p}} \int_{\varepsilon}^{\eta} A^{1-m} \exp \left\{\int_{s}^{\eta}-\frac{N-2}{2 \tau} d \tau\right\} d s \\
& =\frac{\alpha}{N 2^{p-1}} A^{1-m} \eta^{\frac{2-N}{2}}\left(\eta^{N / 2}-\varepsilon^{N / 2}\right),
\end{aligned}
$$

for any $\eta>2^{2 / N} \varepsilon$, we conclude that

$$
-h_{\varepsilon}(\eta) \geq \frac{\alpha}{N 2^{p}} A^{1-m} \eta .
$$

Then for any $\eta>2^{2 / N} \varepsilon$, we have

$$
-f_{\varepsilon}^{\prime}(\eta)=-\eta^{-\frac{p}{2(p-1)}}\left|h_{\varepsilon}(\eta)\right|^{q-2} h_{\varepsilon}(\eta) \geq\left(\frac{\alpha}{N 2^{p}} A^{1-m}\right)^{1 /(p-1)} \eta^{\frac{2-p}{2(p-1)}}
$$

Integrating the above inequality from $2^{2 / N_{\varepsilon}}$ to $\eta$ yields

$$
f_{\varepsilon}\left(2^{2 / N} \varepsilon\right)-f_{\varepsilon}(\eta) \geq \frac{2(p-1)}{p}\left(\frac{\alpha A^{1-m}}{N 2^{p}}\right)^{\frac{1}{p-1}}\left(\eta^{\frac{p}{2(p-1)}}-2^{\frac{p}{N(p-1)}} \varepsilon^{\frac{p}{2(p-1)}}\right),
$$

which implies that

$$
\begin{aligned}
f_{\varepsilon}(\eta) & \leq f_{\varepsilon}\left(2^{2 / N} \varepsilon\right)-\frac{2(p-1)}{p}\left(\frac{\alpha A^{1-m}}{N 2^{p}}\right)^{\frac{1}{p-1}}\left(\eta^{\frac{p}{2(p-1)}}-2^{\frac{p}{N(p-1)}} \varepsilon^{\frac{p}{2(p-1)}}\right) \\
& \leq A-\frac{2(p-1)}{p}\left(\frac{\alpha A^{1-m}}{N 2^{p}}\right)^{\frac{1}{p-1}}\left(\eta^{\frac{p}{2(p-1)}}-2^{\frac{p}{N(p-1)}} \varepsilon^{\frac{p}{2(p-1)}}\right) .
\end{aligned}
$$

Then for any $\eta$ with $\eta^{p / 2(p-1)}>2^{\frac{p}{N(p-1)}} \varepsilon^{\frac{p}{2(p-1)}}+\frac{p}{2(p-1)}\left(\frac{N 2^{p}}{\alpha}\right)^{\frac{1}{p-1}} A^{\frac{m+p-2}{p-1}}$, we have $f_{\varepsilon}(\eta) \leq 0$, it is a contradiction, which means that

$$
\gamma_{\varepsilon} \leq\left(2^{\frac{p}{N(p-1)}} \varepsilon^{\frac{p}{2(p-1)}}+\frac{p}{2(p-1)}\left(\frac{N 2^{p}}{\alpha}\right)^{\frac{1}{p-1}} A^{\frac{m+p-2}{p-1}}\right)^{\frac{2(p-1)}{p}} .
$$

Then the proof is complete.

In what follows, we show several comparison lemmas.

Lemma 2.1. Assume that $0<A_{1} \leq A_{2}, \sigma_{1} \leq \sigma_{2} \leq 0$, and let $\left(f_{1}, h_{1}\right),\left(f_{2}, h_{2}\right)$ be the solutions of the system (1.4) with the initial value conditions $f_{i}(\varepsilon)=$ $A_{i}, h_{i}(\varepsilon)=\sigma_{i}(i=1,2)$. If $f_{1}$ and $f_{2}$ are positive in $\left[\varepsilon, \gamma_{0}\right]$ for some $\gamma_{0}>\varepsilon$, then $f_{1}(\eta) \leq f_{2}(\eta), h_{1}(\eta) \leq h_{2}(\eta)$ for any $\eta \in\left[\varepsilon, \gamma_{0}\right]$.

Proof. If $A_{1}=A_{2}$ and $\sigma_{1}=\sigma_{2}$, then the uniqueness of solution to the initial value problem implies $f_{1}=f_{2}$ and $h_{1}=h_{2}$. Thus, without loss of generality, we may assume that $A_{1}=A_{2}$ and $\sigma_{1}=\sigma_{2}$ do not hold at the same time. 
Firstly, we consider the case $0<f_{1}(\varepsilon)<f_{2}(\varepsilon), h_{1}(\varepsilon)=h_{2}(\varepsilon) \leq 0$. Then we have

$$
\begin{aligned}
h_{2}^{\prime}(\varepsilon)+\frac{N-2}{2 \eta} h_{2}(\varepsilon) & =2^{-p} \beta \eta^{(p-2) / 2(p-1)} f_{2}^{-m}\left|h_{2}\right|^{q-2} h_{2}-\left.\frac{\alpha}{2^{p}} f_{2}^{1-m}\right|_{\eta=\varepsilon} \\
& >2^{-p} \beta \eta^{(p-2) / 2(p-1)} f_{1}^{-m}\left|h_{1}\right|^{q-2} h_{1}-\left.\frac{\alpha}{2^{p}} f_{1}^{1-m}\right|_{\eta=\varepsilon} \\
& =h_{1}^{\prime}(\varepsilon)+\frac{N-2}{2 \eta} h_{1}(\varepsilon),
\end{aligned}
$$

which means that $h_{2}^{\prime}(\varepsilon)>h_{1}^{\prime}(\varepsilon)$. Therefore, there must exist a constant $\delta>\varepsilon$ such that $0<f_{1}<f_{2}, h_{1}<h_{2} \leq 0$ on $(\varepsilon, \delta]$. While if $0<f_{1}(\varepsilon) \leq$ $f_{2}(\varepsilon), h_{1}(\varepsilon)<h_{2}(\varepsilon) \leq 0$. Then $f_{1}^{\prime}(\varepsilon)<f_{2}^{\prime}(\varepsilon)$. Thus, we have the same result as above, that is there exists $\delta>\varepsilon$ such that $0<f_{1}<f_{2}, h_{1}<h_{2} \leq 0$ on $(\varepsilon, \delta]$. Take $\delta_{0}=\max \left\{\eta \in\left(\varepsilon, \gamma_{0}\right] ; 0<f_{1}(\eta) \leq f_{2}(\eta), h_{1}(\eta) \leq h_{2}(\eta) \leq 0\right\}$. Then, clearly, we have $\delta_{0}=\gamma_{0}$, Otherwise, we see that $f_{1}^{\prime}(\eta) \leq f_{2}^{\prime}(\eta)$ for any $\eta \in\left(\varepsilon, \delta_{0}\right]$. Then we have $f_{1}\left(\delta_{0}\right) \leq f_{2}\left(\delta_{0}\right)-f_{2}(\eta)+f_{1}(\eta)$, which means that $f_{1}\left(\delta_{0}\right)<f_{2}\left(\delta_{0}\right)$ since there exists some $\eta$ such that $f_{1}(\eta)<f_{2}(\eta)$. Repeating the above process, we obtain that there exists $\delta>0$ such that $0<f_{1}(\eta) \leq f_{2}(\eta), h_{1}(\eta) \leq h_{2}(\eta) \leq 0$ for $\eta \in\left[\delta_{0}, \delta_{0}+\delta\right)$, which is clearly a contradiction. The proof is complete.

Lemma 2.2. Let $\left(f_{\varepsilon}, h_{\varepsilon}\right)$ be a pair of solution of the problem (1.4), (2.1). Then $f_{\varepsilon}, h_{\varepsilon}$ are both monotone nonincreasing in $\left(\varepsilon, \gamma_{\varepsilon}\right)$; Moreover, for any sufficiently small $\varepsilon_{i}(i=1,2)$ with $0<\varepsilon_{1}<\varepsilon_{2}$, we have $0 \leq f_{\varepsilon_{1}}(\eta)<$ $f_{\varepsilon_{2}}(\eta), h_{\varepsilon_{1}}(\eta)<h_{\varepsilon_{2}}(\eta) \leq 0$ for $\eta \in\left[\varepsilon_{2}, \gamma_{\varepsilon_{1}}\right)$.

Proof. The monotonicity of $f_{\varepsilon}$ is a direct consequence of Proposition 2.1 since $h_{\varepsilon}<0$ in $\left(\varepsilon, \gamma_{\varepsilon}\right)$. So it suffices to consider the case of $h_{\varepsilon}$ with monotonicity. For any sufficiently small $\delta>0$, denote $f_{\delta}(\eta)=f_{\varepsilon}(\eta+\delta), h_{\delta}(\eta)=h_{\varepsilon}(\eta+\delta)$ for $\eta \in\left(\varepsilon, \gamma_{\varepsilon}-\delta\right)$. By Proposition 2.1, we see that

$$
f_{\delta}(\varepsilon)<A, \quad h_{\delta}(\varepsilon)<0 .
$$

Thus by Lemma 2.1, we conclude that

$$
f_{\varepsilon}(\eta+\delta) \leq f_{\varepsilon}(\eta), \quad h_{\varepsilon}(\eta+\delta) \leq h_{\varepsilon}(\eta)
$$

From the arbitrariness of $\delta$, we see that $h_{\varepsilon}$ is monotonic in $\left(\varepsilon, \gamma_{\varepsilon}\right)$.

Furthermore, we note that

$$
f_{\varepsilon_{1}}\left(\varepsilon_{2}\right)<f_{\varepsilon_{1}}\left(\varepsilon_{1}\right)=A=f_{\varepsilon_{2}}\left(\varepsilon_{2}\right), \quad h_{\varepsilon_{1}}\left(\varepsilon_{2}\right) \leq h_{\varepsilon_{1}}\left(\varepsilon_{1}\right)=0=h_{\varepsilon_{2}}\left(\varepsilon_{2}\right),
$$

then by comparison, we conclude that

$$
f_{\varepsilon_{1}}(\eta)<f_{\varepsilon_{2}}(\eta), \quad h_{\varepsilon_{1}}(\eta) \leq h_{\varepsilon_{2}}(\eta), \quad \text { for any } \eta \in\left(\varepsilon_{2}, \gamma_{\varepsilon_{1}}\right)
$$

Thus, we complete the proof.

To obtain the existence of solutions for the problem (1.4)-(1.5) by using the regularized problem, we also need some estimates. 
Lemma 2.3. There exists a constant $C_{0}>0$ independent of $\varepsilon$, such that $\gamma_{\varepsilon}>$ $\varepsilon+C_{0}>4 \varepsilon$. Furthermore, for any $\delta$ with $0<\delta<\min \left\{\frac{\gamma_{\varepsilon}}{2}, \gamma_{\varepsilon}-4 \varepsilon\right\}$, when $p \geq 2$,

$$
f_{\varepsilon}(\eta) \geq A^{\frac{1-m}{p-1}}\left(\frac{\alpha}{N 2^{p}}\right)^{1 /(p-1)} \gamma_{\varepsilon}^{\frac{2-p}{2(p-1)}} \delta, \quad \text { for } \eta \in\left[\varepsilon, \gamma_{\varepsilon}-\delta\right]
$$

when $p<2$,

$$
f_{\varepsilon}(\eta) \geq A^{\frac{1-m}{p-1}}\left(\frac{\alpha}{N 2^{p}}\right)^{1 /(p-1)}\left(\frac{\gamma_{\varepsilon}}{2}\right)^{\frac{2-p}{2(p-1)}} \delta, \quad \text { for } \eta \in\left[\varepsilon, \gamma_{\varepsilon}-\delta\right] .
$$

Proof. We first consider the case $m>1$. Recalling the system (1.4), we see that

$$
h_{\varepsilon}^{\prime}-\frac{2-N}{2 \eta} h_{\varepsilon}=2^{-p} \beta \eta f_{\varepsilon}^{-m} f_{\varepsilon}^{\prime}-\frac{\alpha}{2^{p}} f_{\varepsilon}^{1-m} .
$$

It is equivalent to

$$
\begin{aligned}
\left(h_{\varepsilon} \eta^{(N-2) / 2}\right)^{\prime}= & \frac{\beta}{2^{p}(1-m)} \eta^{N / 2}\left(f_{\varepsilon}^{1-m}\right)^{\prime}-\frac{\alpha}{2^{p}} f_{\varepsilon}^{1-m} \eta^{(N-2) / 2} \\
= & \frac{\beta}{2^{p}(1-m)}\left(\eta^{N / 2} f_{\varepsilon}^{1-m}\right)^{\prime}+\frac{N \beta}{2^{p+1}(m-1)} \eta^{(N-2) / 2} f_{\varepsilon}^{1-m} \\
& -\frac{\alpha}{2^{p}} f_{\varepsilon}^{1-m} \eta^{(N-2) / 2} .
\end{aligned}
$$

Integrating from $\varepsilon$ to $\eta$ yields

$$
\begin{aligned}
-\eta^{(N-2) / 2} h_{\varepsilon}(\eta)= & \frac{\beta}{2^{p}(m-1)}\left(\eta^{N / 2} f_{\varepsilon}^{1-m}-\varepsilon^{N / 2} A^{1-m}\right) \\
& +\left(\frac{2+p \beta}{2(m+p-2)}-\frac{N \beta}{2(m-1)}\right) 2^{-p} \int_{\varepsilon}^{\eta} s^{(N-2) / 2} f_{\varepsilon}^{1-m}(s) d s \\
\leq & \frac{\beta}{2^{p}(m-1)}\left(\eta^{N / 2} f_{\varepsilon}^{1-m}-\varepsilon^{N / 2} A^{1-m}\right) \\
& +\frac{2+p \beta}{N 2^{p}(m+p-2)} f_{\varepsilon}^{1-m}(\eta)\left(\eta^{N / 2}-\varepsilon^{N / 2}\right) \\
& -\frac{\beta}{2^{p}(m-1)} A^{1-m}\left(\eta^{N / 2}-\varepsilon^{N / 2}\right) \\
= & \left(\frac{\beta}{2^{p}(m-1)}+\frac{2+p \beta}{N 2^{p}(m+p-2)}\right) \eta^{N / 2} f_{\varepsilon}^{1-m} \\
& -\frac{\beta}{2^{p}(m-1)} \eta^{N / 2} A^{1-m}-\frac{2+p \beta}{N 2^{p}(m+p-2)} \varepsilon^{N / 2} f_{\varepsilon}^{1-m}(\eta) \\
\leq & \left(\frac{\beta}{2^{p}(m-1)}+\frac{2+p \beta}{N 2^{p}(m+p-2)}\right) \eta^{N / 2} f_{\varepsilon}^{1-m},
\end{aligned}
$$

which implies that

$$
-h_{\varepsilon} \leq\left(\frac{\beta}{2^{p}(m-1)}+\frac{2+p \beta}{N 2^{p}(m+p-2)}\right) \eta f_{\varepsilon}^{1-m} .
$$


Recalling (1.4), we obtain

$$
\begin{aligned}
f_{\varepsilon}^{\prime} & =\eta^{-p / 2(p-1)}\left|h_{\varepsilon}\right|^{q-2} h_{\varepsilon} \\
& \geq-\left(\frac{\beta}{2^{p}(m-1)}+\frac{2+p \beta}{N 2^{p}(m+p-2)}\right)^{1 /(p-1)} \eta^{\frac{2-p}{2(p-1)}} f_{\varepsilon}^{\frac{1-m}{p-1}} .
\end{aligned}
$$

We further have

$$
\left(f_{\varepsilon}^{\frac{m+p-2}{p-1}}\right)^{\prime}(\eta) \geq-\frac{m+p-2}{p 2^{1 /(p-1)}}\left(\frac{\beta}{m-1}+\frac{2+p \beta}{N(m+p-2)}\right)^{1 /(p-1)}\left(\eta^{p / 2(p-1)}\right)^{\prime},
$$

integrating from $\varepsilon$ to $\eta$ yields

$$
\begin{aligned}
f_{\varepsilon}^{\frac{m+p-2}{p-1}}(\eta) \geq & A^{\frac{m+p-2}{p-1}}-\frac{m+p-2}{p 2^{1 /(p-1)}}\left(\frac{\beta}{m-1}+\frac{2+p \beta}{N(m+p-2)}\right)^{1 /(p-1)} \\
& \times\left(\eta^{p / 2(p-1)}-\varepsilon^{p / 2(p-1)}\right) .
\end{aligned}
$$

Take $C_{0}$ by

$$
C_{0}=\frac{1}{2}\left(\frac{1}{2 M_{0}} A^{\frac{m+p-2}{p-1}}\right)^{\frac{2(p-1)}{p}}
$$

where

$$
M_{0}=\frac{m+p-2}{p 2^{1 /(p-1)}}\left(\frac{\beta}{m-1}+\frac{2+p \beta}{N(m+p-2)}\right)^{1 /(p-1)},
$$

then for any $\varepsilon$ with

$$
\varepsilon \leq \frac{1}{6}\left(\frac{1}{2 M_{0}} A^{\frac{m+p-2}{p-1}}\right)^{\frac{2(p-1)}{p}}
$$

we have

$$
f_{\varepsilon}(\eta) \geq 2^{\frac{1-p}{m+p-2}} A>0, \quad \text { for any } \eta \leq C_{0}+\varepsilon,
$$

which implies that $\gamma_{\varepsilon}>C_{0}+\varepsilon>4 \varepsilon$. In addition, recalling (2.4), we see that for any $\eta>2^{2 / N} \varepsilon$,

$$
-f_{\varepsilon}^{\prime}(\eta) \geq\left(\frac{\alpha}{N 2^{p}} A^{1-m}\right)^{1 /(p-1)} \eta^{\frac{2-p}{2(p-1)}}
$$

Then for any $\delta<\min \left\{\frac{\gamma_{\varepsilon}}{2}, \gamma_{\varepsilon}-4 \varepsilon\right\}$, integrating from $\gamma_{\varepsilon}-\delta$ to $\gamma_{\varepsilon}$ yields

$$
f_{\varepsilon}\left(\gamma_{\varepsilon}-\delta\right) \geq \frac{2(p-1)}{p} A^{\frac{1-m}{p-1}}\left(\frac{\alpha}{N 2^{p}}\right)^{1 /(p-1)}\left(\gamma_{\varepsilon}^{\frac{p}{2(p-1)}}-\left(\gamma_{\varepsilon}-\delta\right)^{\frac{p}{2(p-1)}}\right),
$$

which implies

$$
f_{\varepsilon}(\eta) \geq f_{\varepsilon}\left(\gamma_{\varepsilon}-\delta\right) \geq \frac{2(p-1)}{p} A^{\frac{1-m}{p-1}}\left(\frac{\alpha}{N 2^{p}}\right)^{1 /(p-1)}\left(\gamma_{\varepsilon}^{\frac{p}{2(p-1)}}-\left(\gamma_{\varepsilon}-\delta\right)^{\frac{p}{2(p-1)}}\right)
$$


for any $\eta \in\left[\varepsilon, \gamma_{\varepsilon}-\delta\right]$ since $f_{\varepsilon}$ is monotonic on $\left(\varepsilon, \gamma_{\varepsilon}\right)$. Then when $p \geq 2$, we have

$$
f_{\varepsilon}(\eta) \geq A^{\frac{1-m}{p-1}}\left(\frac{\alpha}{N 2^{p}}\right)^{1 /(p-1)} \gamma_{\varepsilon}^{\frac{2-p}{2(p-1)}} \delta
$$

While if $p<2$,

$$
f_{\varepsilon}(\eta) \geq A^{\frac{1-m}{p-1}}\left(\frac{\alpha}{N 2^{p}}\right)^{1 /(p-1)}\left(\frac{\gamma_{\varepsilon}}{2}\right)^{\frac{2-p}{2(p-1)}} \delta .
$$

In what follows, we consider the case $m=1$. Let

$$
A_{0}=\exp \left\{-\frac{2 \alpha}{N \beta}\right\} \text {. }
$$

If $A \leq A_{0}$, we have

$$
\frac{\beta}{2^{p}} \ln A+\frac{\alpha}{N 2^{p-1}} \leq 0
$$

Recalling (1.4), we have

$$
\left(h_{\varepsilon} \eta^{(N-2) / 2}\right)^{\prime}=2^{-p} \beta\left(\eta^{N / 2} \ln f_{\varepsilon}\right)^{\prime}-\frac{\beta N}{2^{p+1}} \eta^{(N-2) / 2} \ln f_{\varepsilon}-2^{-p} \alpha \eta^{(N-2) / 2} .
$$

Integrating from $\varepsilon$ to $\eta$ yields

$$
\begin{aligned}
-h_{\varepsilon} \eta^{(N-2) / 2}= & -\frac{\beta}{2^{p}}\left(\eta^{N / 2} \ln f_{\varepsilon}(\eta)-\varepsilon^{N / 2} \ln A\right) \\
& +\int_{\varepsilon}^{\eta}\left(\frac{\beta N}{2^{p+1}} \ln f(s)+\frac{\alpha}{2^{p}}\right) s^{(N-2) / 2} d s \\
\leq & -\frac{\beta}{2^{p}}\left(\eta^{N / 2} \ln f_{\varepsilon}(\eta)-\varepsilon^{N / 2} \ln A\right) \\
& +\left(\frac{\beta}{2^{p}} \ln A+\frac{\alpha}{2^{p-1} N}\right)\left(\eta^{N / 2}-\varepsilon^{N / 2}\right) \\
\leq & -\frac{\beta}{2^{p}} \eta^{N / 2} \ln f_{\varepsilon}(\eta)+\left(\frac{\beta}{2^{p}} \ln A+\frac{\alpha}{2^{p-1} N}\right) \eta^{N / 2} \\
\leq & -\frac{\beta}{2^{p}} \eta^{N / 2} \ln f_{\varepsilon}(\eta),
\end{aligned}
$$

which implies that

$$
-h_{\varepsilon}(\eta) \leq-\frac{\beta}{2^{p}} \eta \ln f_{\varepsilon}(\eta)
$$

Noting that

$$
f_{\varepsilon}^{\prime}(\eta)=\eta^{-p / 2(p-1)}\left|h_{\varepsilon}\right|^{q-2} h_{\varepsilon}
$$

and $A \leq A_{0} \leq 1$, we arrive at

$$
\begin{aligned}
f_{\varepsilon}^{\prime}(\eta) & \geq-\left(\frac{\beta}{2^{p}}\right)^{\frac{1}{p-1}} \eta^{(2-p) / 2(p-1)}\left|\ln f_{\varepsilon}(\eta)\right|^{\frac{1}{p-1}} \\
& \geq-\left(\frac{\beta}{2^{p}}\right)^{\frac{1}{p-1}} \eta^{(2-p) / 2(p-1)} f_{\varepsilon}^{-\frac{1}{p-1}}(\eta),
\end{aligned}
$$


that is

$$
\left(f_{\varepsilon}^{p /(p-1)}(\eta)\right)^{\prime} \geq-\left(\frac{\beta}{2}\right)^{\frac{1}{p-1}}\left(\eta^{p / 2(p-1)}\right)^{\prime} .
$$

Integrating from $\varepsilon$ to $\eta$ yields

$$
f_{\varepsilon}^{p /(p-1)}(\eta) \geq A^{p /(p-1)}-\left(\frac{\beta}{2}\right)^{\frac{1}{p-1}}\left(\eta^{p / 2(p-1)}-\varepsilon^{p / 2(p-1)}\right) .
$$

Let

$$
C_{0}=\frac{3}{4} 2^{-2(p-1) / p}\left(\frac{2}{\beta}\right)^{2 / p} A^{2} .
$$

Then for any $\varepsilon \leq \frac{1}{3} C_{0}$, we have

$$
f_{\varepsilon}(\eta) \geq \frac{1}{2} A^{p /(p-1)}>0 \text { for any } \eta<C_{0}+\varepsilon,
$$

which means that

$$
\gamma_{\varepsilon}>C_{0}+\varepsilon>4 \varepsilon
$$

While, if $A>A_{0}$, then, by comparison, we see that

$$
\gamma_{\varepsilon}(A) \geq \gamma_{\varepsilon}\left(A_{0}\right)
$$

By taking

$$
C_{0}=\frac{3}{4} 2^{-2(p-1) / p}\left(\frac{2}{\beta}\right)^{2 / p} A_{0}^{2},
$$

we see that the same result holds. In addition, by virtue of (2.4), we see that for any $\eta>2^{2 / N} \varepsilon$,

$$
-f_{\varepsilon}^{\prime}(\eta) \geq\left(\frac{\alpha}{N 2^{p}}\right)^{1 /(p-1)} \eta^{\frac{2-p}{2(p-1)}} .
$$

Integrating from $\eta$ to $\gamma_{\varepsilon}$ yields

$$
f_{\varepsilon}(\eta) \geq \frac{2(p-1)}{p}\left(\frac{\alpha}{N 2^{p}}\right)^{1 /(p-1)}\left(\gamma_{\varepsilon}^{\frac{p}{2(p-1)}}-\eta^{\frac{p}{2(p-1)}}\right) .
$$

Then if $p<2$, we have

$$
f_{\varepsilon}(\eta) \geq f_{\varepsilon}\left(\gamma_{\varepsilon}-\delta\right) \geq\left(\frac{\alpha}{N 2^{p}}\right)^{1 /(p-1)}\left(\frac{\gamma_{\varepsilon}}{2}\right)^{(2-p) / 2(p-1)} \delta,
$$

while if $p \geq 2$,

$$
f_{\varepsilon}(\eta) \geq f_{\varepsilon}\left(\gamma_{\varepsilon}-\delta\right) \geq\left(\frac{\alpha}{N 2^{p}}\right)^{1 /(p-1)} \gamma_{\varepsilon}^{(2-p) / 2(p-1)} \delta
$$

Summing up, we complete the proof. 
Lemma 2.4. Let $0<\varepsilon<\frac{1}{3} C_{0}$. Then for any sufficiently small $\delta>0, f_{\varepsilon}$ is Hölder continuous uniformly and $h_{\varepsilon}$ is Lipschitz continuous uniformly on $\left[\varepsilon, \gamma_{\varepsilon}-\delta\right]$. Namely, for any $\eta_{1}, \eta_{2}$ with $\varepsilon \leq \eta_{1}<\eta_{2} \leq \gamma_{\varepsilon}-\delta$,

$$
\begin{aligned}
& 0 \leq f_{\varepsilon}\left(\eta_{1}\right)-f_{\varepsilon}\left(\eta_{2}\right) \leq M_{1} \delta^{\frac{1-m}{p-1}}\left(\eta_{2}^{\frac{p}{2(p-1)}}-\eta_{1}^{\frac{p}{2(p-1)}}\right), \quad \text { if } m>1 ; \\
& 0 \leq f_{\varepsilon}\left(\eta_{1}\right)-f_{\varepsilon}\left(\eta_{2}\right) \leq M_{2} \delta^{-\frac{1}{p-1}}\left(\eta_{2}^{\frac{p}{2(p-1)}}-\eta_{1}^{\frac{p}{2(p-1)}}\right), \quad \text { if } m=1 \\
& 0 \leq h_{\varepsilon}\left(\eta_{1}\right)-h_{\varepsilon}\left(\eta_{2} \leq \widetilde{C}_{1} \delta^{(1-m p) /(p-1)}\left(\eta_{2}-\eta_{1}\right), \quad \text { if } m>1 ;\right. \\
& 0 \leq h_{\varepsilon}\left(\eta_{1}\right)-h_{\varepsilon}\left(\eta_{2}\right) \leq \widetilde{C}_{2} \delta^{-p /(p-1)}\left(\eta_{2}-\eta_{1}\right), \quad \text { if } m=1,
\end{aligned}
$$

where $M_{1}, M_{2}, \widetilde{C}_{1}, \widetilde{C}_{2}$ are independent of $\varepsilon$.

Proof. We first pay our attention to the case $m>1$. From Lemma 2.3 and the proof of Lemma 2.3, we see that for any $\varepsilon \leq \eta \leq \gamma_{\varepsilon}-\delta$,

$$
\begin{aligned}
-f_{\varepsilon}^{\prime} & \leq\left(\frac{\beta}{2^{p}(m-1)}+\frac{2+p \beta}{N 2^{p}(m+p-2)}\right)^{1 /(p-1)} \eta^{\frac{2-p}{2(p-1)}} f_{\varepsilon}^{\frac{1-m}{p-1}} \\
& \leq \frac{2(p-1)}{p} C \delta^{\frac{1-m}{p-1}}\left(\frac{\beta}{2^{p}(m-1)}+\frac{2+p \beta}{N 2^{p}(m+p-2)}\right)^{1 /(p-1)}\left(\eta^{\frac{p}{2(p-1)}}\right)^{\prime},
\end{aligned}
$$

where $C$ is independent of $\varepsilon$. Then for any $\varepsilon \leq \eta_{1}<\eta_{2} \leq \gamma_{\varepsilon}-\delta$, we have

$$
0 \leq f_{\varepsilon}\left(\eta_{1}\right)-f_{\varepsilon}\left(\eta_{2}\right) \leq M_{1} \delta^{\frac{1-m}{p-1}}\left(\eta_{2}^{\frac{p}{2(p-1)}}-\eta_{1}^{\frac{p}{2(p-1)}}\right),
$$

where

$$
M_{1}=\frac{2(p-1)}{p} C\left(\frac{\beta}{2^{p}(m-1)}+\frac{2+p \beta}{N 2^{p}(m+p-2)}\right)^{1 /(p-1)} .
$$

Moreover, by (1.4), we see that

$$
h_{\varepsilon}^{\prime}=\frac{2-N}{2 \eta} h_{\varepsilon}+2^{-p} \beta \eta^{(p-2) / 2(p-1)} f_{\varepsilon}^{-m}\left|h_{\varepsilon}\right|^{q-2} h_{\varepsilon}-\frac{\alpha}{2^{p}} f_{\varepsilon}^{1-m} .
$$

Thus for any $\varepsilon \leq \eta \leq \gamma_{\varepsilon}-\delta$, when $N \leq 2$, recalling (2.5) and Lemma 2.3, we have

$$
\begin{aligned}
-h_{\varepsilon}^{\prime}(\eta) \leq & C_{0} \frac{2-N}{2} f_{\varepsilon}^{1-m}+2^{-p} C_{0}^{1 /(p-1)} \beta \eta^{p / 2(p-1)} f_{\varepsilon}^{(1-m p) /(p-1)}+\frac{\alpha}{2^{p}} f_{\varepsilon}^{1-m} \\
\leq & C_{1} C_{0} \frac{2-N}{2} \delta^{1-m}+C_{2} 2^{-p} C_{0}^{1 /(p-1)} \beta \eta^{p / 2(p-1)} \delta^{(1-m p) /(p-1)} \\
& +C_{1} \frac{\alpha}{2^{p}} \delta^{1-m},
\end{aligned}
$$

while if $N>2$, we have

$$
\begin{aligned}
-h_{\varepsilon}^{\prime}(\eta) & \leq 2^{-p} C_{0}^{1 /(p-1)} \beta \eta^{p / 2(p-1)} f_{\varepsilon}^{(1-m p) /(p-1)}+\frac{\alpha}{2^{p}} f_{\varepsilon}^{1-m}, \\
& \leq C_{2} 2^{-p} C_{0}^{1 /(p-1)} \beta \eta^{p / 2(p-1)} \delta^{(1-m p) /(p-1)}+C_{1} \frac{\alpha}{2^{p}} \delta^{1-m},
\end{aligned}
$$

where $C_{1}, C_{2}$ are independent of $\varepsilon$, and

$$
C_{0}=\frac{\beta}{2^{p}(m-1)}+\frac{2+p \beta}{N 2^{p}(m+p-2)} .
$$


Summing up, we conclude that

$$
-h_{\varepsilon}^{\prime}(\eta) \leq M_{2} \delta^{(1-m p) /(p-1)} .
$$

In addition, by Lemma 2.2, we see that $-h_{\varepsilon}^{\prime}(\eta)>0$. Thus for any $\varepsilon \leq \eta_{1}<$ $\eta_{2} \leq \gamma_{\varepsilon}-\delta$, we have

$$
0 \leq h_{\varepsilon}\left(\eta_{1}\right)-h_{\varepsilon}\left(\eta_{2}\right) \leq M_{2} \delta^{(1-m p) /(p-1)}\left(\eta_{2}-\eta_{1}\right) .
$$

Next, we turn our attention to the case $m=1$. we see that if $A \leq A_{0} \leq 1$, for any $\varepsilon \leq \eta_{1}<\eta_{2} \leq \gamma_{\varepsilon}-\delta$,

$$
\begin{aligned}
-f_{\varepsilon}^{\prime}(\eta) & \leq\left(\frac{\beta}{2^{p}}\right)^{\frac{1}{p-1}} \eta^{(2-p) / 2(p-1)} f_{\varepsilon}^{-\frac{1}{p-1}}(\eta) \\
& \leq C\left(\frac{\beta}{2^{p}}\right)^{\frac{1}{p-1}} \eta^{(2-p) / 2(p-1)} \delta^{-\frac{1}{p-1}}
\end{aligned}
$$

where $C$ is independent of $\varepsilon$. Then for any $\varepsilon \leq \eta_{1}<\eta_{2} \leq \gamma_{\varepsilon}-\delta$, we have

$$
0 \leq f_{\varepsilon}\left(\eta_{1}\right)-f_{\varepsilon}\left(\eta_{2}\right) \leq \frac{2(p-1)}{p} C\left(\frac{\beta}{2^{p}}\right)^{\frac{1}{p-1}} \delta^{-\frac{1}{p-1}}\left(\eta_{2}^{\frac{p}{2(p-1)}}-\eta_{1}^{\frac{p}{2(p-1)}}\right) .
$$

Moreover, by (1.4), we see that

$$
h_{\varepsilon}^{\prime}=\frac{2-N}{2 \eta} h_{\varepsilon}+2^{-p} \beta \eta^{(p-2) / 2(p-1)} f_{\varepsilon}^{-1}\left|h_{\varepsilon}\right|^{q-2} h_{\varepsilon}-\frac{\alpha}{2^{p}} .
$$

Thus for any $\varepsilon \leq \eta \leq \gamma_{\varepsilon}-\delta$, when $N \leq 2$, recalling (2.9), by using Lemma 2.3 and noticing that $f_{\varepsilon}<A_{0} \leq 1$, we get

$$
\begin{aligned}
-h_{\varepsilon}^{\prime}(\eta) & \leq \frac{\beta(2-N)}{2^{p+1}} f_{\varepsilon}^{-1}+\left(\frac{\beta}{2^{p}}\right)^{p /(p-1)} \eta^{p / 2(p-1)} f_{\varepsilon}^{-p /(p-1)}+\frac{\alpha}{2^{p}} \\
& \leq C_{1} \frac{\beta(2-N)}{2^{p+1}} \delta^{-1}+C_{2}\left(\frac{\beta}{2^{p}}\right)^{p /(p-1)} \eta^{p / 2(p-1)} \delta^{-p /(p-1)}+\frac{\alpha}{2^{p}} ;
\end{aligned}
$$

while if $N>2$, we conclude that

$$
\begin{aligned}
-h_{\varepsilon}^{\prime}(\eta) & \leq\left(\frac{\beta}{2^{p}}\right)^{p /(p-1)} \eta^{p / 2(p-1)} f_{\varepsilon}^{-p /(p-1)}+\frac{\alpha}{2^{p}} \\
& \leq C_{2}\left(\frac{\beta}{2^{p}}\right)^{p /(p-1)} \eta^{p / 2(p-1)} \delta^{-p /(p-1)}+\frac{\alpha}{2^{p}},
\end{aligned}
$$

where $C_{1}, C_{2}$ are independent of $\varepsilon$. Noticing that $\delta$ is sufficiently small, then we have

$$
-h_{\varepsilon}^{\prime}(\eta) \leq \widetilde{C} \delta^{-p /(p-1)},
$$

where $\widetilde{C}$ is independent of $\varepsilon$. In addition, by Lemma 2.2 , we see that $-h_{\varepsilon}^{\prime}(\eta)>0$. Thus for any $\varepsilon \leq \eta_{1}<\eta_{2} \leq \gamma_{\varepsilon}-\delta$, we have

$$
0 \leq h_{\varepsilon}\left(\eta_{1}\right)-h_{\varepsilon}\left(\eta_{2}\right) \leq \widetilde{C} \delta^{-p /(p-1)}\left(\eta_{2}-\eta_{1}\right) \text {. }
$$


While if $A>A_{0}$, recalling (2.8), we see that when $\varepsilon \leq \eta \leq \gamma_{\varepsilon}-\delta$,

$$
\begin{aligned}
-h_{\varepsilon} & \leq\left(\frac{\beta}{2^{p}} \ln \frac{A}{f_{\varepsilon}(\eta)}+\frac{\alpha}{2^{p-1} N}\right) \eta \\
& \leq\left(\frac{\beta}{2^{p}} \ln \frac{A}{C \delta}+\frac{\alpha}{2^{p-1} N}\right) \eta .
\end{aligned}
$$

By (1.4), we see that

$$
-h_{\varepsilon}^{\prime}=-\frac{2-N}{2 \eta} h_{\varepsilon}-2^{-p} \beta \eta^{(p-2) / 2(p-1)} f_{\varepsilon}^{-1}\left|h_{\varepsilon}\right|^{q-2} h_{\varepsilon}+\frac{\alpha}{2^{p}} .
$$

For any $\varepsilon \leq \eta \leq \gamma_{\varepsilon}-\delta$, when $N \leq 2$, by Lemma 2.3, we arrive at

$$
\begin{aligned}
-h_{\varepsilon}^{\prime}(\eta) \leq & \frac{\beta(2-N)}{2^{p+1}} \ln \frac{A}{C \delta}+\frac{\alpha(2-N)}{2^{p} N} \\
& +C \delta^{-1} \frac{\beta}{2^{p}} \eta^{p / 2(p-1)}\left(\frac{\beta}{2^{p}} \ln \frac{A}{C \delta}+\frac{\alpha}{2^{p-1} N}\right)^{1 /(p-1)}+\frac{\alpha}{2^{p}}, \\
\leq & \frac{\beta(2-N)}{2^{p+1}} \ln \frac{A}{C \delta}+\frac{2 \alpha}{2^{p} N}+C \delta^{-1} \frac{\beta}{2^{p}} \eta^{p / 2(p-1)} \\
& \times\left(\frac{\beta}{2^{p}} \ln \frac{A}{C \delta}+\frac{\alpha}{2^{p-1} N}\right)^{1 /(p-1)} ;
\end{aligned}
$$

while if $N>2$, we conclude that

$$
-h_{\varepsilon}^{\prime}(\eta) \leq C \delta^{-1} \frac{\beta}{2^{p}} \eta^{p / 2(p-1)}\left(\frac{\beta}{2^{p}} \ln \frac{A}{C \delta}+\frac{\alpha}{2^{p-1} N}\right)^{1 /(p-1)}+\frac{\alpha}{2^{p}},
$$

where $C$ is independent of $\varepsilon$ and may have some differences. Summing up, we conclude that

$$
-h_{\varepsilon}^{\prime}(\eta) \leq C_{1}|\ln \delta|^{p-1} \delta^{-1}+C_{2} \leq \widetilde{C} \delta^{-p /(p-1)} .
$$

Thus for any $\varepsilon \leq \eta_{1}<\eta_{2} \leq \gamma_{\varepsilon}-\delta$, we have

$$
0 \leq h_{\varepsilon}\left(\eta_{1}\right)-h_{\varepsilon}\left(\eta_{2}\right) \leq \widetilde{C} \delta^{-p /(p-1)}\left(\eta_{2}-\eta_{1}\right),
$$

where $\widetilde{C}$ is independent of $\varepsilon$. The proof is complete.

Lemma 2.5. Let $0<\varepsilon<\frac{1}{3} C_{0}$. For any $\sigma>0$, there exists a constant $\delta>0$ independent of $\varepsilon$ such that

$$
0<f_{\varepsilon}(\eta)<\sigma
$$

for any $\eta \in\left(\gamma_{\varepsilon}-\delta, \gamma_{\varepsilon}\right)$.

Proof. Recalling (2.6), we see that when $m>1$,

$$
\left(f_{\varepsilon}^{\frac{m+p-2}{p-1}}\right)^{\prime}(\eta) \geq-\frac{m+p-2}{p 2^{1 /(p-1)}}\left(\frac{\beta}{m-1}+\frac{2+p \beta}{N(m+p-2)}\right)^{1 /(p-1)}\left(\eta^{p / 2(p-1)}\right)^{\prime} .
$$


Integrating from $\eta$ to $\gamma_{\varepsilon}$ yields

$$
\begin{aligned}
f_{\varepsilon}(\eta) \leq & \left(\frac{m+p-2}{p 2^{1 /(p-1)}}\right)^{\frac{p-1}{m+p-2}}\left(\frac{\beta}{m-1}+\frac{2+p \beta}{N(m+p-2)}\right)^{\frac{1}{m+p-2}} \\
& \times\left(\gamma_{\varepsilon}^{\frac{p}{2(p-1)}}-\eta^{\frac{p}{2(p-1)}}\right)^{\frac{p-1}{m+p-2}} .
\end{aligned}
$$

While if $m=1$, recalling (2.8), we see that

$$
\begin{aligned}
-h_{\varepsilon} & \leq-\frac{\beta}{2^{p}} \eta \ln f_{\varepsilon}(\eta)+\frac{\beta}{2^{p}}\left(\ln A+\frac{2 \alpha}{N \beta}\right) \eta \\
& =\frac{\beta}{2^{p}} \eta \ln \frac{A \exp \{2 \alpha / N \beta\}}{f_{\varepsilon}(\eta)} \\
& \leq \frac{\beta}{2^{p}} \exp \left\{\frac{2 \alpha}{N \beta}\right\} A \eta f_{\varepsilon}^{-1}(\eta) .
\end{aligned}
$$

Therefore, we have

$$
f_{\varepsilon}^{\prime}(\eta) \geq-\left(\frac{A \beta}{2^{p}}\right)^{1 /(p-1)} \exp \left\{\frac{2 \alpha}{N \beta(p-1)}\right\} \eta^{(2-p) / 2(p-1)} f_{\varepsilon}^{-1 /(p-1)}(\eta),
$$

that is

$$
\left(f_{\varepsilon}^{p /(p-1)}\right)^{\prime}(\eta) \geq-2\left(\frac{A \beta}{2^{p}}\right)^{1 /(p-1)} \exp \left\{\frac{2 \alpha}{N \beta(p-1)}\right\}\left(\eta^{p / 2(p-1)}\right)^{\prime} .
$$

Integrating from $\eta$ to $\gamma_{\varepsilon}$ yields

$$
f_{\varepsilon}(\eta) \leq \frac{1}{2}(A \beta)^{1 / p} \exp \left\{\frac{2 \alpha}{N \beta p}\right\}\left(\gamma_{\varepsilon}^{p / 2(p-1)}-\eta^{p / 2(p-1)}\right)^{(p-1) / p} .
$$

By Proposition 2.1 and Lemma 2.3, we see that $C_{0}<\gamma_{\varepsilon}<C_{1}$ is bounded uniformly. Then from the above inequality, it is easy to see that this lemma holds, completing the proof.

\section{The main results and their proofs}

In this section, we shall show the existence and uniqueness of solutions with compact supports, and show the non-existence of solutions without compact support. On the other hands, we also consider the convergent speeds of solutions, and compare the reciprocal of solutions with Dirac function as $t$ tends to infinity in some sense.

Theorem 3.1. For any $\beta \geq 0$ and any $A>0$, there exists at least one solution $(f, h)$ of the initial value problem (1.4)-(1.5). And there exists a positive constant $\gamma$ such that $f(\gamma)=0$, and $f(\eta)>0$ for any $0 \leq \eta<\gamma$.

Proof. Let $\varepsilon<\frac{1}{3} C_{0},\left(f_{\varepsilon}, h_{\varepsilon}\right)$ be the solution of the initial problem (1.4), (2.1). Then by Proposition 2.1, Lemma 2.3, we see that for any $\varepsilon<\frac{1}{3} C_{0}$ there exists a $\gamma_{\varepsilon}$ with $\gamma_{\varepsilon}>C_{0}$ and is bounded uniformly. In addition, by lemma 2.1, we see that $C_{0}<\gamma_{\varepsilon_{1}} \leq \gamma_{\varepsilon_{2}}$ if $0<\varepsilon_{1}<\varepsilon_{2}<\frac{1}{3} C_{0}$. Let

$$
\gamma=\lim _{\varepsilon \rightarrow 0^{+}} \gamma_{\varepsilon} \text {. }
$$


Then $\gamma>0$ exists. In addition, by Lemma 2.2 , we see that $f_{\varepsilon}$ and $h_{\varepsilon}$ are monotone nondecreasing on $\varepsilon$ and bounded for any $\eta \in(0, \gamma)$. Thus $f_{\varepsilon}(\eta), h_{\varepsilon}(\eta)$ converge as $\varepsilon \rightarrow 0^{+}$. For $\eta \in(0, \gamma)$, let

$$
\lim _{\varepsilon \rightarrow 0^{+}} f_{\varepsilon}=f, \quad \lim _{\varepsilon \rightarrow 0^{+}} h_{\varepsilon}=h,
$$

and define

$$
f(0)=A, \quad h(0)=0, \quad f(\gamma)=0 .
$$

We see that $f$ and $h$ are right continuous at 0 from Lemma 2.4 and $f$ is left continuous at $\gamma$ from Lemma 2.5. It follows from Lemma 2.3 that

$$
f(\eta)>0 \text { for any } \eta \in[0, \gamma) .
$$

In addition, by (2.3), we see that for any sufficiently small $\varepsilon_{0}>0$, we have

$$
-h_{\varepsilon}(\eta) \geq \frac{\alpha}{N 2^{p}} A^{1-m} \eta \text { for } \eta \geq \varepsilon_{0},
$$

if $\varepsilon<2^{-2 / N} \varepsilon_{0}$. Thus we have

$$
-h(\eta) \geq \frac{\alpha}{N 2^{p}} A^{1-m} \varepsilon_{0} \quad \text { for } \eta \geq \varepsilon_{0} .
$$

That is $h(\eta)<0$ for $\eta \in(0, \gamma)$. Moreover, Lemma 2.4 implies that $f_{\varepsilon}, h_{\varepsilon}$ are uniformly convergent on $\left[\varepsilon_{0}, \gamma-\delta\right]$ for any sufficiently small $\delta>0$. Combining with (1.4), we see that $f_{\varepsilon}^{\prime}, h_{\varepsilon}^{\prime}$ are uniformly convergent on $\left[\varepsilon_{0}, \gamma-\delta\right]$. Therefore, from the arbitrariness of $\varepsilon_{0}$ and $\delta$, we conclude that $f, h$ are continuously differentiable in $(0, \gamma)$ and satisfy $(1.4)$. Thus $(f, h)$ is a solution of the initial value problem (1.4)-(1.5). The proof is complete.

Theorem 3.2. The problem (1.4)-(1.5) admits at most one solution.

Proof. Assume that $\left(f_{1}, h_{1}\right),\left(f_{2}, h_{2}\right)$ are two solutions of the problem (1.4)(1.5). By the right continuity of $\left(f_{1}, h_{1}\right),\left(f_{2}, h_{2}\right)$, we see that for sufficiently small $\varepsilon>0$, there exist sufficiently small $\delta_{1}>0, \delta_{2}>0$ such that

$$
f_{1}(\varepsilon)=A-\delta_{1}, \quad h_{1}(\varepsilon)=-\delta_{2} .
$$

Define

$$
\widetilde{f}_{2}(\eta)=f_{2}(\eta-\varepsilon), \quad \widetilde{h}_{2}(\eta)=h_{2}(\eta-\varepsilon)
$$

Then

$$
\widetilde{f}_{2}(\varepsilon)=A, \quad \widetilde{h}_{2}(\varepsilon)=0
$$

Thus we have

$$
f_{1}(\eta) \leq \tilde{f}_{2}(\eta)=f_{2}(\eta-\varepsilon), \quad h_{1}(\eta) \leq \widetilde{h}_{2}(\eta)=h_{2}(\eta-\varepsilon) .
$$

Similarly, we also have

$$
f_{2}(\eta) \leq f_{1}(\eta-\varepsilon), \quad h_{2}(\eta) \leq h_{1}(\eta-\varepsilon) .
$$

Summing up, we conclude that for any $\eta \in\left(\varepsilon, \gamma_{1}-\varepsilon\right)$

$$
f_{1}(\eta+\varepsilon) \leq f_{2}(\eta) \leq f_{1}(\eta-\varepsilon), \quad h_{1}(\eta+\varepsilon) \leq h_{2}(\eta) \leq h_{1}(\eta-\varepsilon) .
$$

Recalling Lemma 2.4, and noticing the arbitrariness of $\varepsilon>0$, we finally conclude that $f_{1}(\eta)=f_{2}(\eta), h_{1}(\eta)=h_{2}(\eta)$. The proof is complete. 
Theorem 3.3. Let $(f, h)$ be the solution of the problem (1.4)-(1.5). Then when $m>1$,

$$
f(\eta) \sim o\left((\gamma-\eta)^{\frac{p-1}{m+p-2}}\right)
$$

as $\eta \rightarrow \gamma^{-}$, which means that $\frac{1}{f(s)}$ is integrable on $[0, \gamma]$;

While if $m=1, \frac{1}{f(s)}$ is unintegrable on $[0, \gamma]$ if $p \geq 2$, and is integrable if $p<2$. Furthermore, for any $\sigma>\frac{p-2}{p-1}, \frac{1}{|\ln f(s)|^{\sigma} f(s)}$ is integrable on $[0, \gamma]$ when $m=1, p \geq 2$.

In addition, let

$$
u(x, t)=(t+1)^{-\alpha} f\left((t+1)^{\beta}|x|^{2}\right) \quad \text { with } \beta>0, \alpha=\frac{2+p \beta}{2(m+p-2)}
$$

be the shrinking self-similar solution of Eq. (1.1), and $\varphi(x)$ be a continuous function on $\mathbb{R}^{N}$. Then

(i) if $m>1$, or $m=1$ with $p<2$,

$$
\lim _{t \rightarrow \infty}(t+1)^{\frac{\beta(N(m+p-2)-p)-2}{2(m+p-2)}} \int_{\operatorname{supp} u(\cdot, t)} \frac{\varphi(x)}{u(x, t)} d x=\frac{\pi^{N / 2}}{\Gamma(N / 2)} \kappa \varphi(0),
$$

where

$$
\kappa=\int_{0}^{\gamma} \frac{s^{(N-2) / 2}}{f(s)} d s,
$$

$\Gamma$ is the standard Gamma function. In particular, if $p<N(m+p-2)$, take $\beta=\frac{2}{N(m+p-2)-p}$, then

$$
\lim _{t \rightarrow \infty} \int_{\operatorname{supp} u(\cdot, t)} \frac{\varphi(x)}{u(x, t)} d x=\frac{\pi^{N / 2}}{\Gamma(N / 2)} \kappa \varphi(0) .
$$

(ii) if $m=1$ with $p \geq 2$, then for any small positive constant $\lambda$

$$
\lim _{t \rightarrow \infty}(t+1)^{\frac{N \beta(m+p-2)-(1-\lambda)(2+\beta p)}{2(m+p-2)}} \int_{\operatorname{supp} u(\cdot, t)} \frac{\varphi(x)}{u^{1-\lambda}(x, t)} d x=\frac{\pi^{N / 2}}{\Gamma(N / 2)} \widetilde{\kappa} \varphi(0),
$$

where

$$
\widetilde{\kappa}=\int_{0}^{\gamma} \frac{s^{(N-2) / 2}}{f^{1-\lambda}(s)} d s
$$

$\Gamma$ is the standard Gamma function. Furthermore, if $p<\frac{N(m+p-2)}{1-\lambda}$, take $\beta=\frac{2(1-\lambda)}{N(m+p-2)-p(1-\lambda)}$. Then

$$
\lim _{t \rightarrow \infty} \int_{\operatorname{supp} u(\cdot, t)} \frac{\varphi(x)}{u^{1-\lambda}(x, t)} d x=\frac{\pi^{N / 2}}{\Gamma(N / 2)} \widetilde{\kappa} \varphi(0) .
$$

Proof. Firstly, by system (1.4), we see that if $m>1$, then

$$
\begin{aligned}
\left(h \eta^{(N-2) / 2}\right)^{\prime}= & \frac{\beta}{2^{p}(1-m)}\left(\eta^{N / 2} f^{1-m}\right)^{\prime}+\frac{N \beta}{2^{p+1}(m-1)} \eta^{(N-2) / 2} f^{1-m} \\
& -\frac{\alpha}{2^{p}} f^{1-m} \eta^{(N-2) / 2} .
\end{aligned}
$$


Integrating from $\gamma_{0}=\frac{\gamma}{2}$ to $\eta$ yields

$$
\begin{aligned}
h(\eta) \eta^{(N-2) / 2}= & h\left(\gamma_{0}\right) \gamma_{0}^{(N-2) / 2}-\frac{\beta}{2^{p}(m-1)}\left(\eta^{N / 2} f^{1-m}(\eta)-\gamma_{0}^{N / 2} f^{1-m}\left(\gamma_{0}\right)\right) \\
& +\frac{N \beta-2(m-1) \alpha}{2^{p+1}(m-1)} \int_{\gamma_{0}}^{\eta} s^{(N-2) / 2} f^{1-m}(s) d s
\end{aligned}
$$

Note that

$$
\lim _{\eta \rightarrow \gamma^{-}} \frac{\int_{\gamma_{0}}^{\eta} s^{(N-2) / 2} f^{1-m}(s) d s}{f^{1-m}(\eta)}=0
$$

since that $f(\gamma)=0$. Indeed if $\int_{\gamma_{0}}^{\gamma} s^{(N-2) / 2} f^{1-m}(s) d s$ is bounded, then the above conclusion is obvious; Otherwise, noticing the fact that $h$ is decreasing in $(0, \gamma)$, which means that $f^{\prime}(\gamma)<0$, then by using L'Hôspital's rule, we conclude that

$$
\lim _{\eta \rightarrow \gamma^{-}} \frac{\int_{\gamma_{0}}^{\eta} s^{(N-2) / 2} f^{1-m}(s) d s}{f^{1-m}(\eta)}=\lim _{\eta \rightarrow \gamma^{-}} \frac{\gamma^{(N-2) / 2} f(\eta)}{(1-m) f^{\prime}(\eta)}=0 .
$$

Therefore, we have

$$
\lim _{\eta \rightarrow \gamma^{-}} h(\eta)=-\infty .
$$

Furthermore, recalling (3.1) and combining with (3.2), we arrive at

$$
\lim _{\eta \rightarrow \gamma^{-}} \frac{h(\eta)}{f^{1-m}(\eta)}=-\frac{\beta \gamma}{2^{p}(m-1)} .
$$

Thus for any $0<\varepsilon<\left(\frac{\beta \gamma}{2^{p}(m-1)}\right)^{1 /(p-1)}$, there exists a $\delta>0$ such that when $\gamma-\delta \leq \eta<\gamma$

$$
\left.|| \frac{h(\eta)}{f^{1-m}(\eta)}\right|^{1 /(p-1)}-\left(\frac{\beta \gamma}{2^{p}(m-1)}\right)^{1 /(p-1)} \mid \leq \varepsilon .
$$

Hence, when $\gamma-\delta \leq \eta<\gamma$,

$$
\begin{aligned}
& \frac{f^{\prime}(\eta)}{f^{(1-m) /(p-1)}(\eta)}=-\eta^{-\frac{p}{2(p-1)}} \frac{|h(\eta)|^{1 /(p-1)}}{f^{(1-m) /(p-1)}(\eta)} \\
& \leq-\eta^{-\frac{p}{2(p-1)}}\left(\frac{\beta \gamma}{2^{p}(m-1)}\right)^{1 /(p-1)}+\eta^{-\frac{p}{2(p-1)} \varepsilon} \\
& \frac{f^{\prime}(\eta)}{f^{(1-m) /(p-1)}(\eta)} \geq-\eta^{-\frac{p}{2(p-1)}}\left(\frac{\beta \gamma}{2^{p}(m-1)}\right)^{1 /(p-1)}-\eta^{-\frac{p}{2(p-1)} \varepsilon}
\end{aligned}
$$

Therefore, if $p \neq 2$, we have

$$
\begin{aligned}
& \left(f^{(m+p-2) /(p-1)}\right)^{\prime}(\eta) \leq \frac{2(m+p-2)}{p-2}\left(-\left(\frac{\beta \gamma}{2^{p}(m-1)}\right)^{1 /(p-1)}+\varepsilon\right)\left(\eta^{\frac{p-2}{2(p-1)}}\right)^{\prime}, \\
& \left(f^{(m+p-2) /(p-1)}\right)^{\prime}(\eta) \geq \frac{2(m+p-2)}{p-2}\left(-\left(\frac{\beta \gamma}{2^{p}(m-1)}\right)^{1 /(p-1)}-\varepsilon\right)\left(\eta^{\frac{p-2}{2(p-1)}}\right)^{\prime} .
\end{aligned}
$$


Integrating from $\eta$ to $\gamma$ yields

$$
\begin{aligned}
f^{(m+p-2) /(p-1)}(\eta) \geq & \frac{2(m+p-2)}{p-2}\left(\left(\frac{\beta \gamma}{2^{p}(m-1)}\right)^{1 /(p-1)}-\varepsilon\right) \\
& \times\left(\gamma^{\frac{p-2}{2(p-1)}}-\eta^{\frac{p-2}{2(p-1)}}\right), \\
f^{(m+p-2) /(p-1)}(\eta) \leq & \frac{2(m+p-2)}{p-2}\left(\left(\frac{\beta \gamma}{2^{p}(m-1)}\right)^{1 /(p-1)}+\varepsilon\right) \\
& \times\left(\gamma^{\frac{p-2}{2(p-1)}}-\eta^{\frac{p-2}{2(p-1)}}\right),
\end{aligned}
$$

which implies that

$$
\lim _{\eta \rightarrow \gamma^{-}} \frac{f(\eta)}{(\gamma-\eta)^{\frac{p-1}{m+p-2}}}=\left(\frac{m+p-2}{p-1}\right)^{\frac{p-1}{m+p-2}}\left(\frac{\beta \gamma^{1-\frac{p}{2}}}{2^{p}(m-1)}\right)^{1 /(m+p-2)} .
$$

While if $p=2$, we have

$$
\lim _{\eta \rightarrow \gamma^{-}} \frac{f^{\prime}(\eta)}{f^{1-m}(\eta)}=-\frac{\beta}{4(m-1)} .
$$

Thus for any sufficiently small $0<\varepsilon<\frac{\beta}{4(m-1)}$, there exists a $\delta>0$ such that when $\gamma-\delta \leq \eta<\gamma$,

$$
\begin{aligned}
& \left(f^{m}\right)^{\prime}(\eta) \leq m\left(-\frac{\beta}{4(m-1)}+\varepsilon\right), \\
& \left(f^{m}\right)^{\prime}(\eta) \geq m\left(-\frac{\beta}{4(m-1)}-\varepsilon\right) .
\end{aligned}
$$

Integrating from $\eta$ to $\gamma$ yields

$$
\begin{aligned}
& f^{m}(\eta) \geq m\left(\frac{\beta}{4(m-1)}-\varepsilon\right)(\gamma-\eta), \\
& f^{m}(\eta) \leq m\left(\frac{\beta}{4(m-1)}+\varepsilon\right)(\gamma-\eta) .
\end{aligned}
$$

Thus, we have

$$
\lim _{\eta \rightarrow \gamma^{-}} \frac{f(\eta)}{(\gamma-\eta)^{1 / m}}=\left(\frac{m \beta}{4(m-1)}\right)^{\frac{1}{m}}
$$

Clearly, it coincides with (3.3). According to (3.3), (3.4), we see that $\frac{1}{f(s)}$ is integrable on $[0, \gamma]$ since $m>1$ and $f$ is decreasing.

While if $m=1$, by the equation $f$ and $h$ satisfied, we see that

$$
\left(h \eta^{(N-2) / 2}\right)^{\prime}=2^{-p} \beta\left(\eta^{N / 2} \ln f\right)^{\prime}-\frac{\beta N}{2^{p+1}} \eta^{(N-2) / 2} \ln f-2^{-p} \alpha \eta^{(N-2) / 2} .
$$


Choose $\frac{\gamma}{2} \leq \gamma_{0} \leq \gamma$ and satisfying $f\left(\gamma_{0}\right)<1$, then integrating from $\gamma_{0}=$ to $\eta$ yields

$$
\begin{aligned}
h(\eta) \eta^{(N-2) / 2}= & h\left(\gamma_{0}\right) \gamma_{0}^{(N-2) / 2}+\frac{\beta}{2^{p}}\left(\eta^{N / 2} \ln f(\eta)-\gamma_{0}^{N / 2} \ln f\left(\gamma_{0}\right)\right) \\
& -\frac{N \beta}{2^{p+1}} \int_{\gamma_{0}}^{\eta} s^{(N-2) / 2} \ln f(s) d s-\frac{\alpha}{2^{p}} \int_{\gamma_{0}}^{\eta} s^{(N-2) / 2} d s
\end{aligned}
$$

We first show that

$$
\lim _{\eta \rightarrow \gamma^{-}} \frac{\ln f(\eta)}{\int_{\gamma_{0}}^{\eta} s^{(N-2) / 2} \ln f(s) d s}=\infty .
$$

Noticing that $\ln f(\eta) \rightarrow-\infty$ as $\eta \rightarrow \gamma^{-}$, therefore, if $\int_{\gamma_{0}}^{\eta} s^{(N-2) / 2} \ln f(s) d s$ is bounded, then the above result is proved. Or else, by by using L'Hôspital's rule, we arrive at

$$
\lim _{\eta \rightarrow \gamma^{-}} \frac{\ln f(\eta)}{\int_{\gamma_{0}}^{\eta} s^{(N-2) / 2} \ln f(s) d s}=\lim _{\eta \rightarrow \gamma^{-}} \frac{f^{\prime}(\eta)}{\gamma^{(N-2) / 2} f(\eta) \ln f(\eta)}=\infty
$$

since that $f^{\prime}(\gamma)<0$ and $f(\eta) \ln f(\eta)$ goes to $0^{-}$as $\eta \rightarrow \gamma^{-}$. Therefore, we have

$$
\lim _{\eta \rightarrow \gamma^{-}} \frac{h(\eta)}{\ln f(\eta)}=\frac{\beta \gamma}{2^{p}}
$$

which implies that

$$
\frac{f^{\prime}(\eta)}{|\ln f(\eta)|^{1 /(p-1)}}=-\gamma^{\frac{2-p}{2(p-1)}}\left(\frac{\beta}{2^{p}}\right)^{1 /(p-1)} .
$$

Thus for any sufficiently small $0<\varepsilon<\gamma^{\frac{2-p}{2(p-1)}}\left(\frac{\beta}{2^{p}}\right)^{1 /(p-1)}$, there exists a constant $\delta>0$, such that when $\gamma-\delta \leq \eta<\gamma, f(\eta)<1$ and

$$
\left|\frac{f^{\prime}(\eta)}{|\ln f(\eta)|^{1 /(p-1)}}+\gamma^{\frac{2-p}{2(p-1)}}\left(\frac{\beta}{2^{p}}\right)^{1 /(p-1)}\right|<\varepsilon .
$$

We further have

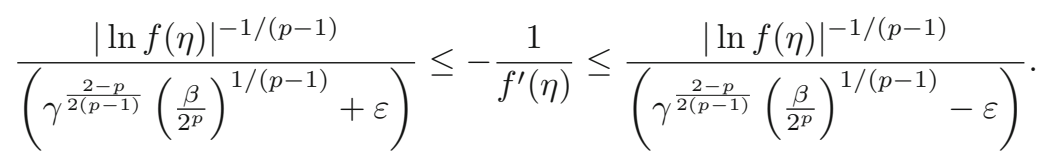

Noticing that

$$
\begin{aligned}
\int_{0}^{\gamma} \frac{1}{f(s)} d s & =\int_{0}^{\gamma-\delta} \frac{1}{f(s)} d s+\int_{\gamma-\delta}^{\gamma} \frac{1}{f(s)} d s \\
& =\int_{0}^{\gamma-\delta} \frac{1}{f(s)} d s+\int_{\gamma-\delta}^{\gamma} \frac{1}{f^{\prime}(s)} d \ln f(s)
\end{aligned}
$$


and

$$
\begin{aligned}
\int_{\gamma-\delta}^{\gamma}|\ln f(s)|^{-1 /(p-1)} d \ln f(s) & =\int_{-\infty}^{\ln f(\gamma-\delta)}|s|^{-1 /(p-1)} d s \\
& =\int_{-\ln f(\gamma-\delta)}^{\infty} s^{-1 /(p-1)} d s .
\end{aligned}
$$

It is easy to see that if $p \geq 2$, the above integral expression is unintegrable, while if $p<2$, then the above integral expression is integrable. Summing up, we conclude that when $m=1, \frac{1}{f(s)}$ is unintegrable on $[0, \gamma]$ if $p \geq 2$, and is integrable if $p<2$. Furthermore, we see that when $p \geq 2$, for any $\sigma>\frac{p-2}{p-1}$,

$$
\int_{\gamma-\delta}^{\gamma} \frac{1}{|\ln f(s)|^{\sigma} f(s)} d s=\int_{\gamma-\delta}^{\gamma}|\ln f(s)|^{-\sigma} \frac{1}{f^{\prime}(s)} d \ln f(s),
$$

and noticing that

$$
\int_{\gamma-\delta}^{\gamma}|\ln f(s)|^{-\sigma}|\ln f(s)|^{-1 /(p-1)} d \ln f(s)
$$

is integrable, which implies that $\frac{1}{|\ln f(s)|^{\sigma} f(s)}$ is integrable on $[0, \gamma]$.

Let $\varphi(x)$ be a continuous function on $\mathbb{R}^{N}$. If $m>1$, or $m=1$ with $p<2$, we note that $\frac{s^{(N-2) / 2}}{f(s)}$ is integrable on $[0, \gamma]$, then we have

$$
\begin{aligned}
\int_{\operatorname{supp} u(\cdot, t)} \frac{\varphi(x)}{u(x, t)} d x & =(t+1)^{\alpha} \int_{\operatorname{supp} u(\cdot, t)} \frac{\varphi(x)}{f\left((t+1)^{\beta}|x|^{2}\right)} d x \\
& =\frac{\pi^{N / 2}}{\Gamma(N / 2)}(t+1)^{\alpha-N \beta / 2} \varphi(\xi) \int_{0}^{\gamma} \frac{s^{(N-2) / 2}}{f(s)} d s \\
& =\frac{\pi^{N / 2}}{\Gamma(N / 2)}(t+1)^{\frac{2+\beta(p-N(m+p-2))}{2(m+p-2)}} \varphi(\xi) \int_{0}^{\gamma} \frac{s^{(N-2) / 2}}{f(s)} d s
\end{aligned}
$$

where $|\xi|<(t+1)^{-\beta / 2} \sqrt{\gamma}$. While if $m=1$ with $p \geq 2$. Since $\frac{1}{|\ln f(s)|^{\sigma} f(s)}$ is integrable on $[0, \gamma]$. Then for any small positive constant $\lambda, \frac{1}{f^{1-\lambda}(s)}$ is integrable on $[0, \gamma]$. Thus, we have

$$
\begin{aligned}
\int_{\operatorname{supp} u(\cdot, t)} \frac{\varphi(x)}{u^{1-\lambda}(x, t)} d x & =(t+1)^{\alpha(1-\lambda)} \int_{\operatorname{supp} u(\cdot, t)} \frac{\varphi(x)}{f^{1-\lambda}\left((t+1)^{\beta}|x|^{2}\right)} d x \\
& =\frac{\pi^{N / 2}}{\Gamma(N / 2)}(t+1)^{\alpha(1-\lambda)-N \beta / 2} \varphi(\xi) \int_{0}^{\gamma} \frac{s^{(N-2) / 2}}{f^{1-\lambda}(s)} d s \\
& =\frac{\pi^{N / 2}}{\Gamma(N / 2)}(t+1)^{\frac{(2+\beta p)(1-\lambda)-N \beta(m+p-2)}{2(m+p-2)}} \varphi(\xi) \int_{0}^{\gamma} \frac{s^{(N-2) / 2}}{f^{1-\lambda}(s)} d s,
\end{aligned}
$$

where $|\xi|<(t+1)^{-\beta / 2} \sqrt{\gamma}$. Up to now, we complete the proof.

Theorem 3.4. The Eq. (1.3) admits no solution without compact support, namely, there is no $f(\eta) \in C^{2}(0,+\infty)$ such that $f(\eta)>0$ for any $\eta \in[0,+\infty), f$ and $\eta^{(p-2) / 2}\left|f^{\prime}\right|^{p-2} f^{\prime}$ are right continuous at 0 , and $f$ satisfies (1.3) in $(0,+\infty)$. 
Proof. If $p \geq 2$, let $h=\eta^{p / 2}\left|f^{\prime}\right|^{p-2} f^{\prime}$. Then $h$ is continuously differentiable in $(0,+\infty), h$ is right continuous at 0 with $h(0)=0$, and Eq. (1.3) is transformed to system (1.4). From (1.4), we get that

$$
h^{\prime}=\frac{2-N}{2} \frac{h}{\eta}+2^{-p} \beta \eta^{(p-2) / 2(p-1)} f^{-m}|h|^{q-2} h-\frac{\alpha}{2^{p}} f^{1-m} .
$$

Noticing that $\lim _{\eta \rightarrow 0^{+}} h^{\prime}(\eta)=\lim _{\eta \rightarrow 0^{+}} \frac{h}{\eta}$, therefore, $\lim _{\eta \rightarrow 0^{+}} h^{\prime}(\eta)$ exists, and $h^{\prime}(0)=-\frac{\alpha}{2^{p-1} N} A^{1-m}<0$. While if $p<2$, let $g=\eta^{(p-1) / 2}\left|f^{\prime}\right|^{p-2} f^{\prime}$. Then $g$ is continuously differentiable in $(0,+\infty)$, and $g$ is right continuous at 0 with $g(0)=0$. A direct calculation yields

$$
g^{\prime}+\frac{N-1}{2} \frac{g}{\eta}=\frac{\beta}{2^{p}}|g|^{q-2} g-\frac{\alpha}{2}^{-p} \eta^{-1 / 2} f^{1-m}(\eta) .
$$

Note that $g(0)=0$, then we have

$$
\lim _{\eta \rightarrow 0^{+}} g^{\prime}(\eta)=-\infty
$$

which means that $\eta^{-1 / 2} h$ is decreasing near 0 . All in all, there exists a $\eta_{0}>0$ such that for any $0<\eta<\eta_{0} h(\eta)<h(0)=0$ and $f^{\prime}(\eta)<0$ for $0<\eta<\eta_{0}$, thus we have

$$
h\left(\eta_{0}\right)<h(0), \quad f\left(\eta_{0}\right)<f(0)=A .
$$

Consider the problem (1.4) with the following initial condition

$$
\tilde{f}\left(\eta_{0}\right)=A, \quad \widetilde{h}\left(\eta_{0}\right)=0 .
$$

By Proposition 2.1, we see that there exists a $\gamma_{0}$ such that

$$
\tilde{f}\left(\gamma_{0}+\eta_{0}\right)=0 .
$$

Then by comparison, we see that

$$
f\left(\gamma_{0}\right) \leq \tilde{f}\left(\gamma_{0}+\eta_{0}\right)=0
$$

which is a contradiction.

\section{References}

[1] Allen, L.J.S.: Persistence and extinction in single-species reaction-diffusion models. Bull. Math. Biol. 45, 209-227 (1983)

[2] Friedman, A., McLeod, B.: Blow-up of solutions of nonlinear degenerate parabolic equations. Arch. Rational Mech. Anal. 96, 55-80 (1986)

[3] Wiegner, M.: Blow-up for solutions of some degenerate parabolic equations. Differ. Integral Eqs. 7, 1641-1647 (1994)

[4] Bertsch, M., Bisegna, P.: Blow-up of solutions of a nonlinear parabolic equation in damage mechanics. Eur. J. Appl. Math. 8, 89-123 (1997)

[5] Angenent, S.: On the formation of singularities in the curve shortening flow. J. Differ. Geom. 33, 601-633 (1991) 
[6] Dal Passo, R., Luckhaus, S.: A degenerate diffusion problem not in divergence form. J Differ. Equ. 69, 1-14 (1987)

[7] Leray, J.: Sur le mouvement d'un liouide visqueux emplissant l'espace. Acta Math. 63, 193-248 (1934)

[8] Gilding, B.H., Peletier, L.A.: On a class of similarity solutions of the porous media equation. J. Math. Anal. Appl. 55, 351-364 (1976)

[9] Gilding, B.H., Peletier, L.A.: On a class of similarity solutions of the porous media equation II. J. Math. Anal. Appl. 57, 522-538 (1977)

[10] Gilding, B.H.: On a class of similarity solutions of the porous media equation III. J. Math. Anal. Appl. 77, 381-402 (1980)

[11] Escobedo, M., Kavian, O.: Variational problems related to self-similar solutions of the heat equation. Nonlinear Anal. TMA 11, 1103-1133 (1987)

[12] Naito, Y., Suzuki, T.: Radial symmetry of self-similar solutions for semilinear heat equations. J. Differ. Equ. 163, 407-428 (2000)

[13] Wang, C.P., Yin, J.X.: Shrinking self-similar solutions of a nonlinear diffusion equation with nondivergence form. J. Math. Anal. Appl. 289, 387-404 (2004)

[14] Giga, Y., Kohn, R.: Asymptotically self-similar blow-up of semilinear heat equations. Commun. Pure Appl. Math. 38, 297-319 (1985)

[15] Jin, C.H., Yin, J.H.: Periodic solutions of a non-divergent diffusion equation with nonlinear sources. Discrete Continuous Dyn. Syst. B 17, 101-126 (2012)

Chunhua Jin and Jingxue Yin

School of Mathematical Sciences

South China Normal University

Guangzhou 510631

China

e-mail: jinchhua@126.com

Received: 29 January 2012.

Accepted: 10 July 2012. 